\title{
Journal of large-scale
}

research facilities

Journal of large-scale research facilities, 2, A56 (2016)

http://dx.doi.org/10.17815/jlsrf-2-81

Published: 21.04.2016

\section{CXS: Coherent X-ray scattering at the UE49-SGM at BESSY II}

Helmholtz-Zentrum Berlin für Materialien und Energie *

Instrument Scientists:

- Dr. W. Dieter Engel, Helmholtz-Zentrum Berlin für Materialien und Energie, phone: +49 (0) 30 8062-14380, e-mail: dietrich.engel@helmholtz-berlin.de

- Dr. Durga Mishra, Helmholtz-Zentrum Berlin für Materialien und Energie, phone: +49 (0) 30 8062-14379, e-mail: durgamadhab.mishra@helmholtz-berlin.de

- Prof. Dr. Stefan Eisebitt, Technische Universität Berlin, and Max-Born-Institut für Nichtlineare Optik und Kurzzeitspektroskopie im Forschungsverbund Berlin e.V., phone: +49 (0) 30 6392-1300, e-mail: eisebitt@ mbi-berlin.de

\begin{abstract}
The coherent soft-x-ray scattering experiment CXS has been developed to study nano-structured magnetic and nonmagnetic thin film samples in transmission or reflection geometry. A nanometer precision movable sample stage in a 1 Tesla magnet vector field together with a movable CCD detector, variable in sample - CCD distance, allows both XMCD and XMLD experiments in transmission and reflection as well as imaging techniques such as Fourier transform holography and ptychography.
\end{abstract}

\section{1 introduction}

The CXS end station (see Figure 1) at UE49-SGM (Helmholtz-Zentrum Berlin für Materialien und Energie, 2016) allows utilizing the high coherent photon flux from a BESSY II low-beta undulator for $\mathrm{x}$-ray interference based experiments. The CXS is optimized for membrane window samples (typ. $\mathrm{Si}_{3} \mathrm{~N}_{4}, \mathrm{MgO}$ or glass) covered by functional layer systems with thicknesses between $1-400 \mathrm{~nm}$ and lateral structure sizes from few $\mathrm{nm}$ to several $\mu \mathrm{m}$. The sample itself can be in size between $2 \times 2$ and $10 \times 10 \mathrm{~mm}^{2}$ in size. The sample holder consists of two rotatable parts, one is the sample holder itself with the option to rotate the sample by $340^{\circ}$ and to connect the sample electrically. The second part is a yoke-like rotator with four slots for sensors, e.g. a photo diode, also rotatable by $340^{\circ}$ relative to the sample holder.

The Cartesian parallel kinematic (Noll et al., 2009) based sample table with absolute encoders enables the precise movement of the sample relative to the focus by $\pm 5 \mathrm{~mm}$ with $20 \mathrm{~nm}$ accuracy. An additional

\footnotetext{
${ }^{*}$ Cite article as: Helmholtz-Zentrum Berlin für Materialien und Energie. (2016). CXS: Coherent X-ray scattering at the UE49-SGM at BESSY II. Journal of large-scale research facilities, 2, A56. http://dx.doi.org/10.17815/j1srf-2-81
} 
multi-window membrane unit can be positioned in front of the sample holder containing different gold masks, pinholes and zone plates to optionally further shape and control the sample illumination.
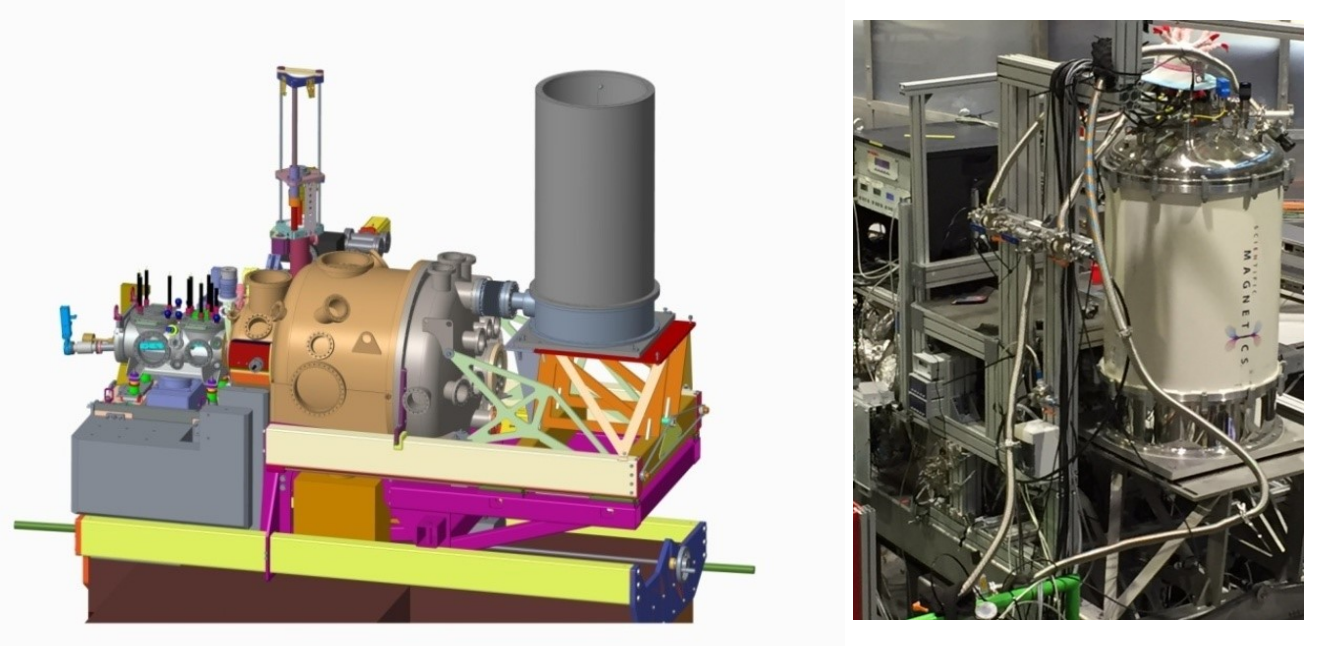

Figure 1: Coherent soft x-ray scattering end station at the UE49-SGM.

The setup is very flexible in its scattering geometry via an in vacuum movable CCD detector, as shown in Figure 2, which allows experiments to be performed in both transmission and reflection. The detector is a Princeton CCD, back illuminated with a 2048 x 2048 pixel chip and $13.5 \mu \mathrm{m}$ pixel size. In front of the CCD an aluminum foil is attached to absorb the visible stray light. In front of the foil, a slider with four windows is mounted, holding several beamstops, which can be moved relative to the CCD.

Due to the operation at a low beta undulator in conjunction with few optical element beamline design of the UE49-SGM beamline, the coherent flux is exceptionally high. Coherence parameters of the illumination can be adjusted in situ. X-ray magnetic dichroism can be used for spectroscopy as well as for spectroscopic contrast in scattering or imaging experiments via the availability of a $1 \mathrm{~T}$ magnetic vector field at the sample and full polarization control of the soft x-ray beam. The 3D magnetic vector field is achieved in a large bore superconducting magnet with $100 \mathrm{~mm}$ clear aperture in the incident beam direction, enabling the flexible scattering geometries and sample motion mentioned above.

All internal and external parts of the CXS and the main parts of the beamline are controlled by a LabView based computer software, including automatic scan programs, control of the super-conducting electromagnet and sample transfer. 


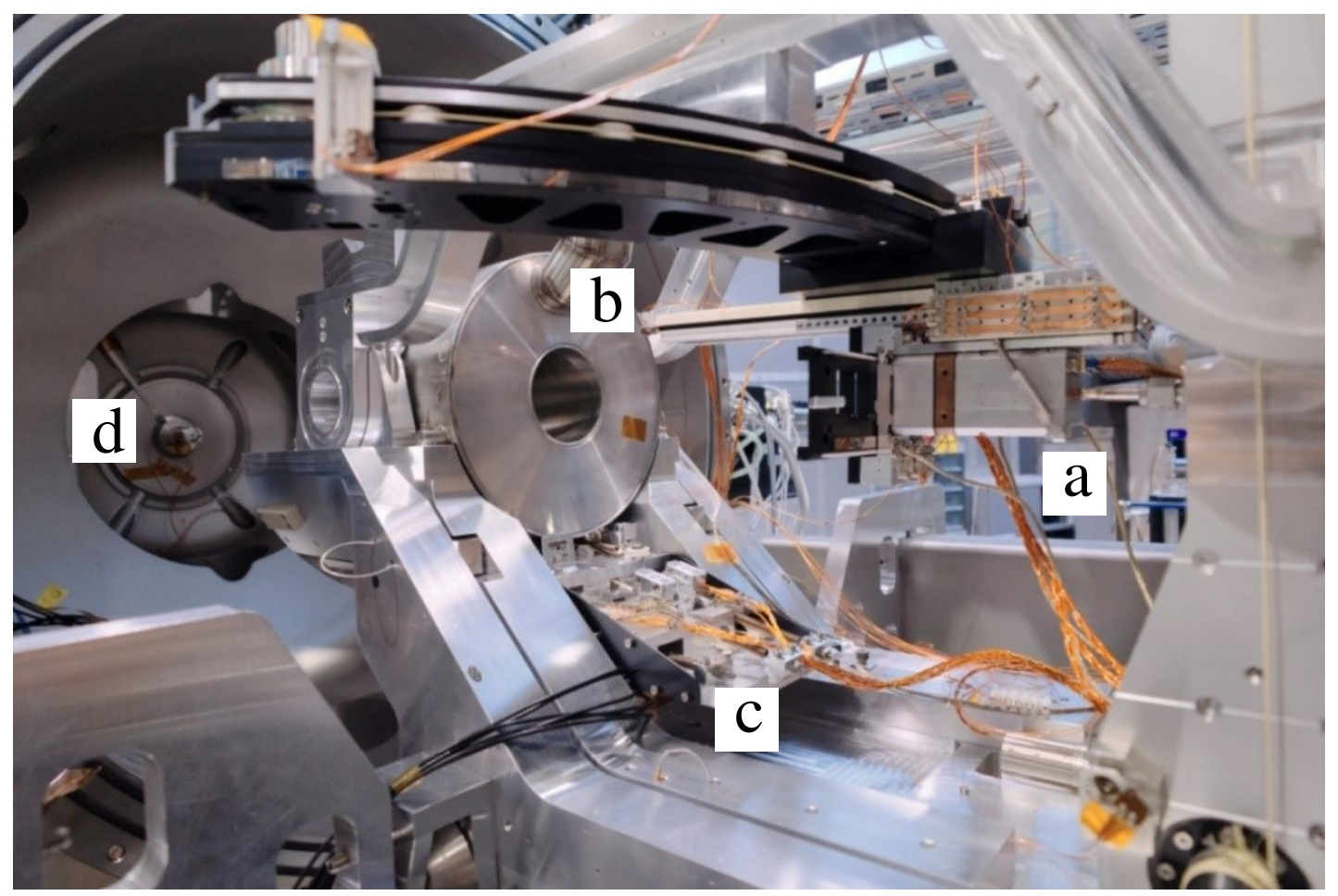

Figure 2: In vacuum view showing the detector unit (a), the magnet (b), partly the sample stage (c) and the differential pumping stage (d).

\section{Typical applications}

All experiments are performed at a base pressure of $10^{-7}$ mbar and room temperature. It is planned for 2016 to implement a cryostat. All samples can be connected electrically via up to 16 terminals and can be measured in a $1 \mathrm{~T}$ magnetic vector field.

a) Imaging

- Fourier transform holography (FTH) in transmission and reflection (future feature)

- Scanning FTH in transmission

- Ptychography in transmission

- Spectro-microscopy of nanostructures

b) Scattering

- XMCD, XMLD

- Soft X-ray resonant magnetic scattering (SXRMS)

- Soft x-ray GISAXS

- Reflectometry

- Small angle x-ray scattering at normal and oblique incidence

c) Spectroscopy

- X-ray absorption spectroscopy

- XMCD, XMLD 


\section{Sample environment}

- Sample holder

- Variable for reflection and transmission

- 16 electronic connectors

- Cryostat (future feature)

- Beam shaping unit

- Pinholes

- Zone plates

- Double slits

- Masks for FTH

- Magnetic field of 1T vectorial

\section{Technical data}

\begin{tabular}{|l|l|}
\hline Location & 10.1 \\
\hline Source & UE49 \\
\hline Monochromator & Spherical VLS grating monochromator \\
\hline Energy range & $90-1400 \mathrm{eV}$ \\
\hline Energy resolution & Up to 12000 \\
\hline Polarization & Full polarization control \\
\hline Focus size & $1 \mathrm{x} 4 \mu \mathrm{m}$ \\
\hline Divergence & $18 \mathrm{mrad}$ vertical, 26 mrad horizontal \\
\hline Longitudinal coherence & Control via monochromator \\
\hline Transversal coherence & Control via apertudes and sample position relative to the focus \\
\hline Flux & $7 \mathrm{x} 10^{14}$ photons/s/100mA/0.1\% BW \\
\hline Sample-CCD detector distance & Variable: $27-52 \mathrm{~cm}$ \\
\hline Sample movement & xyz-stage: $\pm 5 \mathrm{~mm}$ in all directions with $20 \mathrm{~nm}$ accuracy \\
\hline Sample rotation & $340^{\circ}$ \\
\hline Diode section rotation & $340^{\circ}$ \\
\hline CCD rotation around the sample & $\pm 20^{\circ}$ up/down and left/right \\
\hline
\end{tabular}

\section{References}

Helmholtz-Zentrum Berlin für Materialien und Energie. (2016). The UE49 SGM RICXS beamline at BESSY II. Journal of large-scale research facilities, 2, A54. http://dx.doi.org/10.17815/j1srf-2-78

Noll, T., Holldack, K., Reichardt, G., Schwarzkopf, O., \& Zeschke, T. (2009). Parallel kinematics for nanoscale Cartesian motions. Precision Engineering, 33(3), 291 - 304. http://dx.doi.org/10.1016/j.precisioneng.2008.07.001 